only for multiple myeloma. Moreover, as the authors acknowledge, analyses of various cohorts of radiation workers (which overlap in part with the cohort studied by Kneale and Stewart) in the United States and elsewhere ${ }^{34}$ find no evidence of statistical incompatibility between the cancer risks associated with such occupational exposure and those found in the survivors of the Japanese atomic bombs. ${ }^{6}$ Consequently, we think that little weight should be attached to the results of the paper of Kneale and Stewart.

MARK LITTLE CHRIS SHARP Medical Department National Radiological Protection Board Chilton, Didcot OX11 ORQ

1 Kneale GW, Stewart AM. Factors affecting recognition of cancer risks of nuclear workers. Occup Environ Med 1995;52:515-23.

2 Gilbert ES, Omohundro E, Buchanan JA, Holter NA. Mortality of workers at the Hanford

3 Kendall GM, Muirhead CR, MacGibbon BH, et al. Mortality and occupational exposure to et al. Mortality and occupational exposure to
radiation: first analysis of the National radiation: first analysis of the National
Registry for Radiation Workers. BMf 1992; Registry for
304:220-5.

4 Cardis E, Gilbert ES, Carpenter L, et al. Effects of low doses and low dose rates of external ionizing radiation: cancer mortality among nuclear industry workers in three countries. Radiat Res 1995;142:117-32.

5 Cox DR, Hinkley DV. Theoretical statistics. London: Chapman and Hall, 1974.

6 Shimizu Y, Kato H, Schull WJ. Studies of the mortality of A-bomb survivors. 9. Mortality, 1950-85: Part 2. Cancer mortality based on 1950-85: Part 2. Cancer mortality based on the recently revised

Author's reply-Most of the difficulties which Little and Sharp have with our paper would seem to stem from a failure to understand that our statistical methods were simply practical applications of the general theory of nested likelihood ratio tests. This theory is a very general method of identifying statistical tests that have a wide range of optimum properties provided one has a sufficiently general statistical model of a parametric kind that describes how the data might have been generated, and provided this model satisfies certain easily checkable mathematical restrictions. Why this is so can be discovered in any general text book of mathematical statistics, either the one referenced in our paper-namely, Kendall and Stuart ${ }^{1}$ - or the one preferred by Little and Sharp-namely, Cox and Hinkley. ${ }^{2}$

For studying the relation between radiation and carcinogenesis relevant parametric models can be found in Breslow and Day. ${ }^{3}$ For example, how intuitive ideas about a case-control study can be justified by making use of a parametric model is shown in Vol I page 248, and two formulae for parametric models for cohort studies are given in Vol II page 186. The choice of which formula depends upon whether the cohort study is progressing in continuous time (first formula) or in discrete units of time (second formula). We naturally used the second formula as our radiation doses were only available as annual totals.

The mathematical techniques and limited computer facilities available in 1980 did not allow direct (case-control) methods to be used in cohort studies. Therefore, Vol II of Breslow and Day deals mainly with studies where various approximations (such as Poisson regression when person-years at risk can be calculated) lead to the standard procedures recommended by Little and Sharp.
But although we made use of the formulae on page 186, our numerical methods (for completing the necessary maximum likelihood calculations) differed from the ones recommended by Breslow and Day over 15 years ago.

If new variations of standard methods were not allowed there would be little progress in science. Nevertheless, as we agree with our critics that any new methodology should have its results checked against standard methods, we included in our paper two tables expressly for this purpose. Thus table 7, which includes the basic nested likelihood ratio tests and parameter estimates by maximum likelihood, shows that the most important of the three extra variables influencing relations between radiation and carcinogenesis was exposure age. This is important because virtually all of our models which had included an optimised minimum value for critical exposure age were significant at the $5 \%$ or $1 \%$ level, whereas the models without an optimised value for this variable had much lower levels of significance. Then comes table 9, which shows the results of testing the null hypothesis of no radiation effect by a method exactly equivalent to the standard one recommended by Little and Sharp, but uses the windowed doses prescribed by optimised values of minimum critical exposure age and minimum critical latency. Consequently the significance levels in this table (which lay between $1 \%$ and $0.1 \%$ ) and which were calculated by the standard formulae, had greater strength than the ones in table 7, as they did not take account of the window restrictions.

It should also be noted that although in two papers which used the standard methods approved by Little and Sharp, ${ }^{45}$ lip service was paid to the idea that latency was important-by lagging for doses at stated intervals - this was a very crude method of estimating optimal latency compared with the one we used.

Having explained the theory behind our methodology, it is time to turn to the more detailed criticisms of the Little and Sharp. It is true that in our analysis the critical minimums for exposure age and latency were confined to values of integral years as, with dose estimates only available for whole years, we were forced to use the discrete time formula of Breslow and Day. However, with maximum likelihood as the method of risk estimation, the integral year estimate would necessarily have a slightly lower maximum than any fractional year estimates for continuous doses. Therefore, as our calculated level of significance was based on integral years, it provided a less rigid test of the null hypothesis than one based on daily or weekly doses, and thus left us "playing safe".

Little and Sharp's criticism of the degrees of freedom estimate for a window which allows a zero estimate of effective dose, shows some confusion in their minds about the standard tabular $\chi^{2}$ (which has many degrees of freedom for testing independence in tabulated data) and the $\chi^{2}$ corresponding to generalised deviance in the nested likelihood ratio theory. The degrees of freedom for this $\chi^{2}$ depend not on the number of cells in some mythical table containing all the relevant data, but on the number of variables being estimated. The situation is exactly analogous to the formulae used by Gilbert $e t$ $a l^{4}$ and Cardis et $a l^{5}$ for calculating the significance of their results based on lagged dose estimates. By this method some workers with recorded doses were left with an effective dose of zero. But this no more altered the approximate normal distribution of their test statistic, than it would have altered the approximate $\chi^{2}$ distribution arising in the nested likelihood ratio theory.

Finally, for anyone interested in this arena of statistical theory, it may be of interest to know that the theory of nested likelihood ratio tests was discovered by combining the standard (tabular) $\chi^{2}$ with the standard analysis of variance ratio of $\chi^{2} s$, and that was how $\chi^{2}$ came to be relevant. All this can be investigated either in Kendall and Stuart or in Cox and Hinkley.

GEORGE W KNEALE ALICE M STEWART

Department of Public Health and Epidemiology, University of Birmingham,

1 Kendall MG, Stuart A. The advanced theory of statistics. Vol 1-3. London: Charles Griffin, 1963.

2 Cox DR, Hinkley DV. Theoretical statistics. London: Chapman and Hall, 1964.

3 Breslow NE, Day NE. Statistical methods in cancer research. Vol I. The analysis of casecontrol studies. Vol II. The design and analysis of cohort studies. LARC Sci Publ 1980;32:186.

4 Gilbert ES, Omohundro E, Buchanan JA, Holter NA. Mortality of workers at the Hanford site: 1945-86. Health Phys 1993;64: 577-90.

5 Cardis E, Gilbert ES, Carpenter L, et al. Effects of low doses and low dose rates of external ionizing radiation: cancer mortality among nuclear industry workers in three among nuclear industry workers in

Parental exposure to radiation and childhood cancer

This study ${ }^{1}$ was referred to by Roman et al (p 78) as a "study linking dosimetry information contained within the National Registry of Radiation Workers (NRRW) with records of childhood cancer held in the National Registry of Childhood Tumours (NRCT)". This statement needs amplification. From the outset, the Oxford Survey of Childhood Cancers (OSCC) has been a partner in this project. Identifying particulars for some 40000 parents have been abstracted from the interview records of the OSCC; these data are not contained within the files of the National Registry of Childhood Tumours.

TOM SORAHAN University of Birmingham, Institute of Occupational Health, Edgbaston, Birmingham B13 2TT

1 Kendall GM, Muirhead CR, Draper GJ. Parental exposure to radiation and childhood cancer. Radiological Protection Bulletin 1993, 147:7-13.

2 Eve Roman, Pat Doyle, Pat Ansell, Diana Bull, Valerie Beral. Health of children born to medical radiographers. Occup Environ Med 1996;53:73-9.

\section{Offspring sex ratio as an indicator of reproductive hazards}

Editor-In a recent letter the sex ratio was discussed as an indicator of occupational exposures. ${ }^{12}$

The sex ratio at birth is a prevalence measure reflecting the sex programming at the time of conception and the survival until birth. The sex ratio has been suggested to reflect the hormone concentration at the time of conception. ${ }^{134}$ According to this hypothesis high levels of gonadotropin changes the ratio towards more girls. External exposures may have this effect as 